\title{
SOPHIE BAVA, ROUTES MIGRATOIRES ET ITINÉRAIRES RELIGIEUX. DES SÉNÉGALAIS MOURIDES ENTRE TOUBA ET MARSEILLE
}

Paris, Panafrika, 2017, 432 p.

\section{Anouk Cohen}

Éditions de l'EHESS | «Archives de sciences sociales des religions »

$2019 / 4 n^{\circ} 188 \mid$ pages 262 à 264

ISSN 0335-5985

Article disponible en ligne à l'adresse :

https://www.cairn.info/revue-archives-de-sciences-sociales-desreligions-2019-4-page-262.htm

Distribution électronique Cairn.info pour Éditions de l'EHESS.

(C) Éditions de l'EHESS. Tous droits réservés pour tous pays.

La reproduction ou représentation de cet article, notamment par photocopie, n'est autorisée que dans les limites des conditions générales d'utilisation du site ou, le cas échéant, des conditions générales de la licence souscrite par votre établissement. Toute autre reproduction ou représentation, en tout ou partie, sous quelque forme et de quelque manière que ce soit, est interdite sauf accord préalable et écrit de l'éditeur, en dehors des cas prévus par la législation en vigueur en France. Il est précisé que son stockage dans une base de données est également interdit. 


\section{Archives de sciences sociales des religions}

188 | octobre-décembre 2019

Bulletin bibliographique

Sophie BAVA, Routes migratoires et itinéraires religieux. Des Sénégalais mourides entre Touba et Marseille

Paris, Panafrika, 2017, 432 p.

\section{Anouk Cohen}

\section{OpenEdition}

Journals

Édition électronique

URL : http://journals.openedition.org/assr/48469

ISSN : $1777-5825$

Éditeur

Éditions de l'EHESS

Édition imprimée

Date de publication : 5 décembre 2019

Pagination : 262-264

ISBN : 9782713227844

ISSN : 0335-5985

Référence électronique

Anouk Cohen, «Sophie вAvA, Routes migratoires et itinéraires religieux. Des Sénégalais mourides entre Touba et Marseille », Archives de sciences sociales des religions [En ligne], 188 | octobre-décembre 2019, mis en ligne le 08 janvier 2022, consulté le 02 décembre 2019. URL : http://journals.openedition.org/ assr/48469

Ce document a été généré automatiquement le 2 décembre 2019.

(C) Archives de sciences sociales des religions 


\section{Sophie BAVA, Routes migratoires et itinéraires religieux. Des Sénégalais mourides entre Touba et Marseille}

Paris, Panafrika, 2017, 432 p.

\section{Anouk Cohen}

\section{RÉFÉRENCE}

Sophie BAVA, Routes migratoires et itinéraires religieux. Des Sénégalais mourides entre Touba et Marseille, Paris, Panafrika, 2017, 432 p.

1 Plus de dix ans après avoir soutenu sa thèse, Sophie Bava a fait l'effort de revenir sur ce travail pour restituer les résultats d'une recherche alors innovante sur la manière dynamique et réciproque dont les expériences migratoires reconfigurent les expériences religieuses. À partir d'une méthode d'enquête multi-localisée entre Touba, la ville sainte mouride, et Marseille, enrichie par une lecture remarquablement maitrisée de la littérature confrérique, son ouvrage examine comment se recompose un espace mouride à Marseille. La description détaillée des délocalisations et des négociations auxquelles la mobilité de certains mourides donne lieu met en exergue comment ces modalités de circulation soutiennent des constructions religieuses transnationales. Les portraits de fidèles qui jalonnent les trois parties de l'ouvrage permettent de saisir en quoi concrètement les migrations, par leur nature même, ravivent et transforment le « croire » mouride (p. 21) en prenant appui sur des réseaux économiques, religieux et imaginaires ainsi que sur la construction d'espaces religieux repensés en migration comme les dahiras (associations mourides) et les «maisons Sérigne Touba ».

2 Les premières pages sont consacrées à une présentation éclairante des études mourides du siècle passé qui ont fait la part belle à l'activité économique, politique et sociale de la confrérie au détriment de sa vie religieuse. Ce constat a conduit Sophie Bava à porter 
l'attention au rapport quotidien, ordinaire et intime des fidèles au mouridisme, deuxième confrérie soufie sunnite au Sénégal (après la Tijanyyia). Étudiée au prisme de la migration, cette relation est examinée du point de vue de sa recomposition « en train de se faire». Dans ce but, l'auteure commence par retracer l'itinéraire de Cheikh Amadou Bamba, le fondateur de la confrérie. Cet «exercice "d'anthropologie prophétique" appliquée » (p. 81) permet au lecteur de mieux cerner, aux chapitres suivants, comment les fidèles puisent constamment dans le corpus mouride pour adapter, voire transplanter leur religion au gré de leur mobilité.

3 La seconde partie entre dans le vif du sujet en retraçant les itinéraires religieux, économiques, familiaux et sociaux des migrants mourides. Après un état de l'art du débat sur les migrations qui permet de positionner la thèse de l'ouvrage dans le champ, le rappel de l'histoire des circulations sénégalaises à Marseille met en exergue l'inscription de la mobilité mouride dans une histoire longue de relations commerciales et religieuses entre le Sénégal et Marseille, autrefois étape du parcours en bateau vers la Mecque. Cette contextualisation permet de mieux saisir les enjeux actuels de l'articulation des activités économiques et religieuses mourides dans le processus migratoire. À partir de trois portraits de personnalités saillantes illustrant le fonctionnement des dahira-s à Marseille, l'auteure montre comment s'y développent des activités commerciales qui transforment et recomposent l'éthos mouride, alors profondément axé sur la valeur travail. Contrairement aux chercheurs spécialistes de cette confrérie qui se sont principalement intéressés à la façon dont l'éthique mouride s'adapte à la rationalité économique, Sophie Bava analyse comment s'invente et se construit une autre manière d'être mouride, qui procède du dynamisme de l'entreprise économique migrante et de la négociation avec le local qu'elle induit. Dans ce but, l'auteure décrit comment la réforme de la dahira par la hiérarchie confrérique convertit cet espace initialement prioritairement économique en un espace également religieux. Car si la dahira occupe une place centrale dans l'activité commerciale mouride à Marseille, pour l'élite religieuse des marabouts (maitres spirituels et temporels) elle n'est pas une structure assez solide pour soutenir la survivance et la vigueur de la tradition mouride à l'étranger. Afin d'appuyer le fonctionnement du réseau cultuel, un des fils de Cheikh Amadou Bamba, mobilisé par la confrérie sur les liens avec la migration, fonde les «maisons Sérigne Touba ». Créées pour accueillir les «cheikh-s itinérants " et organiser les ziyara (visites religieuses au cheikh), ces maisons sont conçues comme une "projection de l'espace de Touba dans le territoire de la migration à travers le transfert des problématiques religieuses et sociales qui font vivre la confrérie, une projection des événements mourides dans de nouveaux petits espaces sacrés » (p.198). L'installation croissante de maisons Sérigne Touba reconfigure également l'espace mouride mondialisé qui s'appuie désormais sur une dynamique à la fois religieuse et économique.

4 La troisième partie (la plus riche) consacrée à l'étude de la recomposition de l'expérience mouride entre Touba et Marseille approfondit cette analyse en examinant plus en détail les "mécanismes de déterritorialisation du "croire" » (p. 201). Y sont particulièrement étudiés les "bricolages en train de se faire " (en référence à la démarche d'André Mary), de manière à saisir les modalités de formation d'un « dispositif religieux migrant » entre Marseille et Touba. Pour mieux cerner l'enjeu de ces bricolages, l'auteure commence par décrire le paysage religieux dans lequel s'insère le mouridisme en France et à Marseille. À rebours des approches socio-politiques abordant la question de l'islam de France au prisme du schéma «intégration/ 
exclusion", l'auteure appréhende "l'islam vécu» (p. 213). Ce changement de paradigme prend appui sur l'analyse croisée des pratiques religieuses et migratoires pour mettre au jour le fonctionnement en mouvements du croire mouride en veillant à souligner aussi bien les changements et les nouveautés que les permanences et les prolongements. Cette démarche s'inscrit nécessairement dans une approche historique et comparative (Paris, Marseille, Sénégal) complétée par l'examen du corpus religieux afin d'explorer la construction par les mourides migrants d'une légitimation religieuse de leur mobilité à partir des récits de l'exil de Cheikh Amadou Bamba. Cependant, analyse l'auteure, en migrant, les mourides de la première génération cherchent moins à revivre l'expérience de leur cheikh qu'à se sentir proche de lui en veillant à contribuer à la grandeur de Touba. Dans leur esprit, l'effort, le travail et les gains financiers qui en résultent sont voués à revenir à la ville sainte. Ce devoir qui donne sens à la mobilité est la difficulté majeure de l'expérience migratoire. Pour la surmonter, les fidèles puisent dans l'ethos mouride: «Travailler premièrement pour pouvoir être un bon mouride, travailler pour gagner sa vie, travailler pour construire Touba, travailler la pratique religieuse et travailler pour organiser le mouridisme en migration » (p. 250). Afin de les soutenir par la création de dahira-s, des descendants indirects de Cheikh Amadou Bamba à Marseille se disputent l'autorité. Les conflits opposent les anciens commerçants qui souvent ont migré sans leur famille aux plus jeunes installés en France avec leur famille et aux intellectuels mourides qui, tout en défendant un lien étroit à la ville sainte de Touba, soutiennent l'idée d'une ouverture de la confrérie sur le monde en créant notamment des espaces de transmission pour les enfants nés en France. Cependant, tous confèrent à la migration un sens commun : « Le seul départ ne compte pas ou peu, le Pèlerinage à Touba peut par exemple renforcer le mythe [...]. Autour du migrant mouride et des symboles qu'il réveille, un nouveau territoire mouride fait d'articulations entre de petits morceaux d'espaces, prend forme et prend sens au gré des ré-interprétations religieuses » (p. 255). Ce sont ces manières plurielles de s'unir à la confrérie et à Touba depuis Marseille que l'auteure explore dans la suite de l'ouvrage. "Pour que le dispositif religieux migre [...] tout passe par des négociations » (ibid). Mais les stratégies élaborées par les fidèles pour s'inscrire dans la lignée croyante doivent toujours être soumises à la validation de la hiérarchie confrérique dont chaque verdict ravive les rivalités entre acteurs. À ceux mentionnés plus haut, s'ajoutent la société locale et les enfants de migrants nés en France dont les réactions ont une incidence directe sur les prises de décision. La langue employée lors des conférences (wolof ou français) cristallise les désaccords et révèle différentes façons d'être un mouride dans la migration. Une fois de plus néanmoins, les différents projets religieux de la confrérie s'appuient sur une même synergie: la visite des cheikh-s. En effet, les fidèles recherchent moins les réponses dans les textes, qu'ils n'attendent qu'elles ne soient livrées par la bouche de leur cheikh accueilli dans la dahira. «Le cheikh est comme un parent pour le taalibé, il vient le voir et lui transmet la baraka, il le guide lorsqu'il a de grandes décisions à prendre dans sa vie (parfois même par le téléphone) et reçoit de l'argent en échange de ses services» (p. 300). Aux yeux des fidèles, l'itinérance du cheikh augmente sa valeur spirituelle. Ainsi, parmi les descendants légitimes de Cheikh Amadou Bamba, Sérigne Mourtada Mbacké, fondateur des "maisons Sérigne Mourtada» est celui qui se présente comme le "véritable entrepreneur officiel du mouridisme en migration » (p. 281). Car il a su répondre aux réalités de ses taalibé-s exilés en venant à eux. Loin de seulement maintenir une proximité entre la hiérarchie et ses taalibé-s, la visite des cheikh-s contribue à 
construire un territoire mouride à Marseille et à en délimiter des frontières tangibles. La circulation d'objets rituels, en particulier les images pieuses de Cheikh Amadou Bamba et de ses petits-fils ainsi que les cassettes audio et vidéo des récits du fondateur, est centrale dans la constitution de cet espace. Elles permettent de se projeter dans une relation forte et intime à la confrérie et à Touba. Ainsi, l'auteure montre comment tout un dispositif religieux est mis en place par la circulation d'hommes et d'objets qui recréent une continuité territoriale entre les différents lieux de la migration.

Comme la continuation de la relation du taalibé au cheikh est fondamentale dans la migration réussie du dispositif religieux, le travail, selon la doctrine mythique fidèlement appliquée par le mouride migrant, est constitutif de la recomposition de son identité religieuse. L'auteure examine comment, au fil des années, un glissement s'est opéré entre les catégories " travail » et " argent ». Ainsi, aujourd'hui, c'est davantage par l'argent redistribué aux dahira-s que les taalibé-s maintiennent le lien à la confrérie et à Touba, en dépit du rejet du monde matériel évoqué par Cheikh Amadou Bamba dans ses écrits. Le pèlerinage dans la ville sainte dit le grand magal de Touba est un autre élément majeur de l'élaboration du dispositif religieux migrant. Selon l'auteure, ce " "lieu-moment" mystique» (p. 319) annuel institué au xxe siècle pour commémorer l'exil du fondateur en est la véritable matrice. Sophie Bava distingue deux manières d'être mouride migrant et pèlerin : "Celle où l'on célèbre l'événement sans pèlerinage effectif, mais qui reste tout de même une forme de voyage, un pèlerinage affectif peutêtre, et celle du pèlerinage au véritable sens du terme » (p. 320). Le premier type de pèlerinage s'effectue dans le dahira où il est célébré collectivement par des centaines de taalibés. Pendant que les hommes prient, les femmes aidées des enfants cuisinent. À ce moment de sociabilité religieuse intense, toutes les discussions et les prières convergent vers Touba. La ville sainte paraît alors proche à ceux qui restent. Sophie Bava a aussi porté l'attention sur les mourides qui partent en suivant, étape par étape, le départ depuis Marseille de quelques pèlerins mourides pour étudier ce qui se joue dans cet " "entre deux" de la construction religieuse ». Elle montre que Touba est le moment privilégié pour le migrant qui cherche à réaffirmer sa foi et montrer sa réussite en finançant son voyage, aidant sa famille restée au Sénégal, accueillant d'autres pèlerins dans une concession acquise à Touba grâce aux gains de l'exil ou encore en contribuant au financement de projets collectifs dans la ville, telle que la rénovation de la Grande mosquée ou la construction d'un hôpital. La visibilité du migrant et de son utilité pour le groupe atteint à cet instant son paroxysme. Consacré à la création de la fédération des mourides du Sud de la France, le dernier chapitre met en exergue les jeux de pouvoir suscités par les négociations des mourides avec la société locale. Plus globalement, cette étude de cas permet, écrit l'auteure, de repenser « le pacte laïque à la française » (p. 202).

6 Finalement, ce sont les modalités de la mondialisation d'une confrérie entre proximité (se sentir proche de Cheikh Amadou Bamba et de Touba grâce à la visite des cheikh-s et aux objets qui circulent, etc.) et transnationalisation (mobilités des cheikh-s et des taalibé-s, nouvelles technologies de la communication et de l'information qui permettent de maintenir le lien avec la lignée croyante, etc.) que l'ouvrage permet de documenter. Ainsi, à travers les croisements entre religion et migration, c'est une autre approche de l'islam que Sophie Bava dessine: éloignée des représentations et plus proche des pratiques sociales et cultuelles. Aussi, elle propose de considérer la 
migration moins comme une rupture que comme une ressource dans les constructions religieuses. 